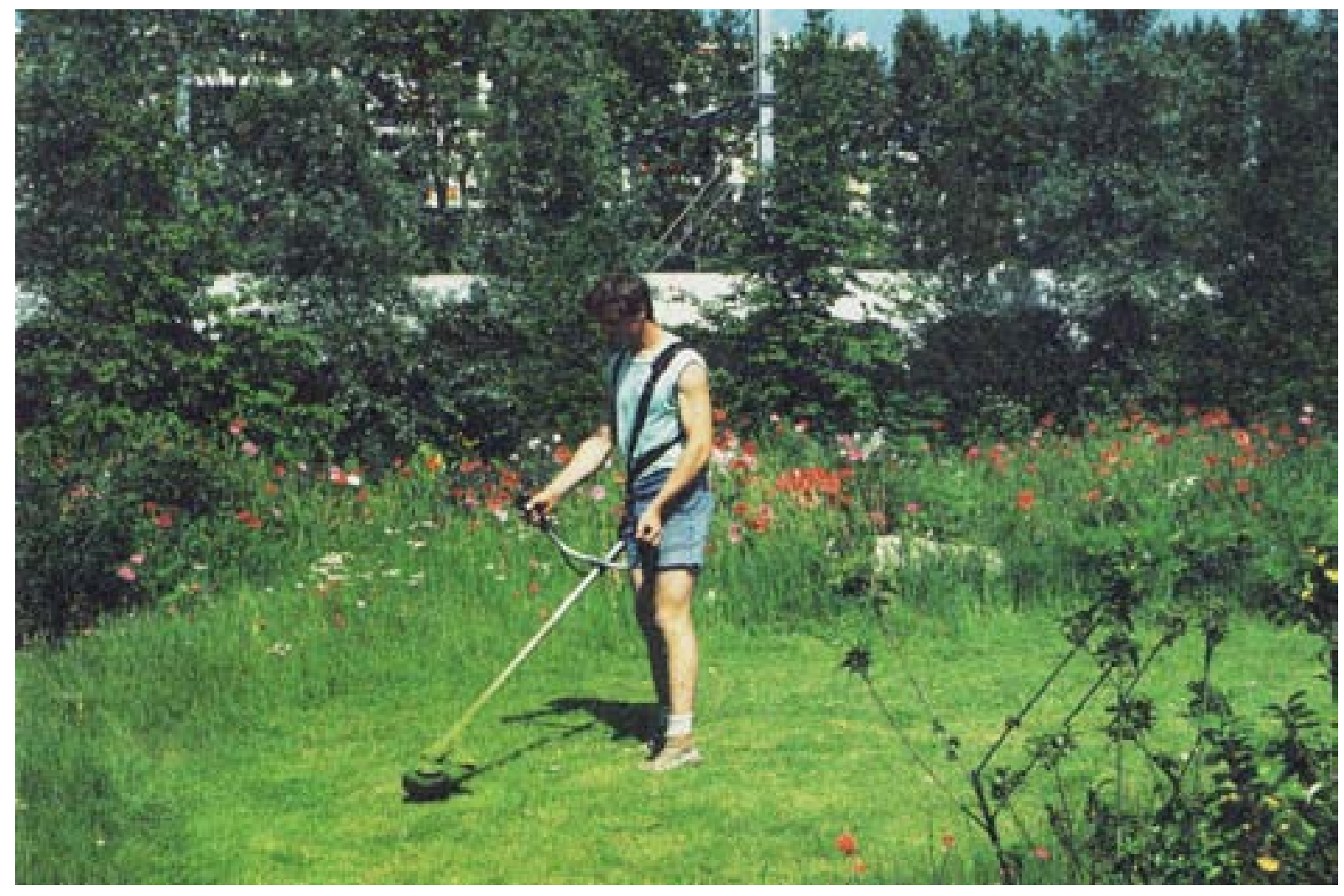




\section{La naturaleza}

\section{como material de construcción Ophélia Mantz Rafael Beneytez}

La consideración de la estructura de sistemas, de acontecimientos relacionales y de ciclos temporales observados en los seres vivos siempre ha sido una fuente de inspiración para el hombre. La naturaleza, como sistema abierto nos ofrece la oportunidad de aprender tanto de los resultados como de sus procesos. La construcción de nuevos paradigmas inspirados en la naturaleza será el hilo argumental del artículo: la naturaleza como material de construcción de nuevos modelos de pensamiento y de organización dentro del campo de la arquitectura y de la ciudad.

\section{PALABRAS CLAVE}

Contingencia, Ecología temporal, Cronobiología, Agroforestería, ecología técnica KEYWORDS

Contingency, Temporal Ecology, Chronobiology, Agroforestry, Technical Ecology

LA CONTINGENCIA EN ARQUITECTURA: ECOLOGÍA TEMPORAL Y TÉCNICA
La aparición de la agricultura 9000 años antes de nuestra era, junto con la Revolución industrial del siglo XIX, han marcado rupturas muy importantes de la relación del hombre con la naturaleza. El Homo Sapiens se desarrolló en adaptación a su medio hasta el momento en que supo dominar las técnicas de la agricultura. Desde la llamada "Revolución Neolítica", el Hombre comenzó a adaptar el entorno a sus necesidades. En otras palabras, la evolución de nuestra especie hoy, gracias a sus capacidades cognitivas, ha dejado de ser esencialmente biológica para ser cultural. El hombre, desde la Revolución Industrial, en lugar de utilizar recursos producidos por la biosfera, dentro de ciclos temporales

\begin{abstract}
Ophélia Mantz
Metz, Francia, 1977. Arquitecta titulada por la Escuela Superior de Arquitectura de Paris-Belleville, UP8, Francia; Master en Arquitectura Bioclimática y sostenibilidad por la Escuela Técnica Superior de Arquitectura de Madrid. Actualmente es Visiting Assistant Professor y coordinadora de la asignatura de proyectos para el primer año de Bachelor en la Texas Tech University, College of Architecture en Lubbock, Texas, USA. Codirectora de Z4Z4 AAA con Rafael Beneytez, con quien dirige la Oficina Z4Z4 AAA (Architects Activities Associated) como unidad productiva de diseño. La línea de investigación de su trabajo se desarrolla entorno a la construcción del pensamiento ecológico en el campo de la arquitectura y la ciudad.
\end{abstract}

\section{Rafael Beneytez Durán}

Madrid, España, 1972. PhD por el DPA ETSAM dirige Z4Z4-Z4A. Es actualmente Associate Professor of the College of Architecture at Texas Tech University. Ha sido Profesor de Proyectos Arquitectónicos del DPA de la ETSAM - UPM y profesor de Proyectos y Estética y Composición de la ESAyT. Ha sido invitado por las Escuelas de Arquitectura de Architectural Association, NJIT New Jersey, TTU Texas, A\&M Texas, UPC Barcelona, ETSAV El Vallés, Granada, Segovia, Londres, Würzburg, Münster, Oporto, Delft, Limerick o Harvard, para impartir conferencias y talleres. Su trabajo de investigación se centra en explorar la arquitectura como condición y no como objeto, y se apoya fundamentalmente en el trabajo teórico de Gilbert Simondon y las nuevas sensibilidades del discurso arquitectónico sobre la atmósfera como forma, abordando el problema como pregunta estética desde la técnica y en la cultura. La obra del estudio que dirigen ha sido publicada y premiada nacional e internacionalmente por entidades tales como Architectural Review, Mark, ARQ, BIEAU, COAM, Europan, Bienal de Estambul.

Fig. 01

Fotografía de Gilles Clément, Parc Citroën, Le jardín en Mouvement. 
correspondientes a su contemporaneidad, ha recurrido masivamente a aquellas otras materias ligadas a la litosfera. Estos recursos, como son el carbono, el gas, el petróleo o los sedimentos son productos de ecosistemas desaparecidos hace cientos de millones de años. La capacidad del hombre por adecuar la naturaleza a sus requerimientos, le ha llevado a desentenderse de los lazos de dependencia que tiene con ella. Por ello, el hombre se ha desligado de los ciclos temporales que le correspondían. Esta acelerada carrera de consumo de materias limitadas, nos ha llevado a una hipertrofia del presente. La amnesia por identificar nuestros orígenes nos impide proyectarnos colectivamente hacia un futuro de algo mas de unos años'.

Sin necesidad de recurrir a una situación de sumisión a una naturaleza mitificada, hay que reconocerse en la urgencia por reinventar las bases de un nuevo "contrato natural", tema ya introducido por el filósofo francés Michel Serres en 1990².

La naturaleza, como sistema abierto, al que pertenecemos, es y ha sido siempre una fuente de inspiración. El conocimiento de los seres vivos, construido desde la observación, nos permite entender con cada vez mayor precisión, la complejidad de los sistemas que albergan. Sus dinámicas permanentes de evolución, de adaptación, de emergencia así como su tendencia a la multiplicidad de variaciones y su abanico de soluciones redundantes e infinitas, nos ofrecen la oportunidad de aprender tanto de los resultados como de sus procesos.

En el campo de la biología, el concepto de autopoiesis ${ }^{3}$ nos ayuda a repensar nuestros sistemas de desarrollo desde la aceptación de la contingencia, las cooperaciones, las alianzas, la simbiosis que se presentan como los principales factores de emergencia e innovación dentro del mundo de los seres vivos. La naturaleza ofrece flujos de energía y de materia optimizados a través de dinámicas de cooperación entre actores gracias a la información que intercambian. El biomimetismo, concepto desarrollado por Janine Benyus en 1998, propone ir más allá de lo ya conocido por imitar la Naturaleza. Defiende la observación e investigación sobre las formas y estructuras naturales (soluciones de los organismos vivos en situaciones de mutación) con la ambición de impulsarnos a preparar transformaciones venideras y necesarias en la esfera de la economía ligada a los cambios globales que afectan a los ecosistemas.

La consideración de la estructura de sistemas, de acontecimientos relacionales y de ciclos temporales observados en los seres vivos, es parte del hilo argumental de este artículo que, en correspondencia con el título de la convocatoria añade: la naturaleza como material de construcción "de nuevos modelos de pensamiento dentro del campo de la arquitectura y de la ciudad".

Para defender este argumento, por una parte hemos recurrido al concepto de exaptación ${ }^{4}$ desarrollado en Paleobiología. Este concepto se refiere a cómo los organismos vivos en determinados contextos utilizan órganos preexistentes de un modo diferente du su estado original. La mutación y adaptación son dos asuntos que revelan bien el concepto subyacente de la contingencia. Como tal concepto, la contingencia ilustra bien las posibilidades de un nuevo cambio de paradigma que se expresa con claridad en la obra de Gilles Clément y que podría promover el papel fundador de la contingencia en arquitectura.

Por otra parte, se pone de manifiesto la necesidad de reconsiderar la técnica, empleada por el hombre actuando en la naturaleza, 
para lograr dicha contingencia. Junto a ambas se propone una mirada de admiración y mayor valoración del conocimiento técnico de la naturaleza, para reconsiderar la ecología desde una concepción tecnológica ${ }^{5}$ que restaura los lazos entre la humanidad y el resto de los seres vivos.

GILLES

CLÉMENT Y LA

CONTINGENCIA:

HACIA UN CAMBIO

DE PARADIGMA
El periodo histórico así como la localización geográfica elegida para enfocar el hilo argumental del texto quieren defender un acto constituyente dentro del pensamiento ecológico en Francia: París, 1980-1995. En Europa, la Alemania del siglo XIX empieza a construir una pensamiento ecológico cuestionando la relación del hombre con la naturaleza. La influencia de los viajes de Alexander Von Humboldt en América del sur en 1804 así como la creación del término Ökologie ${ }^{6}$ por parte del biólogo y naturalista alemán Ernst Haeckel (1834-1919) son las primicias del activismo político alemán que nació después de la segunda Guerra Mundial. Sin embargo en Francia, la toma de consciencia que permitió reconsiderar el lazo del hombre con la naturaleza fue mucho más tardío. En los años 1930, Bernard Charbonneau empezó a establecer un pensamiento ecológico a través de su tesis Le sentiment de la nature. Force Révolutionnaire que según Thierry Paquot ${ }^{7}$ fue el primer texto de ecología política en Francia. En este país europeo, el periodo de post guerra fue marcado por un suceso de acontecimientos históricos que reflejan la voluntad de una sociedad que quiso reestructurarse en profundidad. En 1958 nació la quinta republica y diez años más tarde el movimiento estudiantil de mayo de 1968 abrió un periodo de profundo cuestionamiento de la sociedad francesa dando por consecuencia el nacimiento de nuevas políticas. Nuevos valores culturales se fueron legitimando como por ejemplo la reconsideración del papel del medio natural dentro de las políticas de planeamiento territorial${ }^{8}$.

Entre los años 1970 y 1990, numerosos acontecimientos políticos ${ }^{9}$ lograron construir una redefinición de la noción de paisaje, dentro de la opinión pública, así como en el campo de la filosofía y de la arquitectura. Durante estos años coinciden, por una parte la creación de un nuevo departamento de filosofía en la Universidad París VIII'10 capitaneado entre otros por Jacques Derrida, Gilles Deleuze, Bruno Latour, Félix Guattari y Michel Serres, quienes influyeron el pensamiento de Gilles Clément. Por otra, la creación de l'École Nationale Supérieure du Paysage en Versalles en 1976 que empezó a formar numerosos paisajistas DPLG" como Gilles Clément, capaces de difundir una nueva mirada sobre la noción de paisaje.

Este marco histórico de vanguardia, considerado como constituyente en la evolución del pensamiento ecológico en Francia, marcó toda una generación a la que pertenecen Gilles Clément (1943), Jean Nouvel (1945) y Dominique Perrault (1953).

La postura técnica y estética de Gilles Clément, jardinero y paisajista francés en su relación con la naturaleza ha cambiado los paradigmas de observación en numerosos proyectos de arquitectura en París y en Francia. Ejemplos como el edificio de la Biblioteca Nacional de Francia construida por Dominique Perrault así como la Fundación Cartier de Jean Nouvel ilustran bien los cambios de paradigmas que generó el pensamiento de Gilles Clément. Con el fin de poder esbozar conceptos ligados al mundo de los seres vivos, es decir al de la biología. La primera 
Fig. $\mathrm{O} 2$

Fotografía de Gilles Clément, Parc Citroën, Le jardín en Mouvement.

Fig. 03

Île de la Vassivière, Gilles Clément.

línea directora de Gilles Clément en los años 90, fue aquella que, apoyada en el concepto del Tiers-paysage o Tercer Paisaje, promovió el giro a este nuevo paradigma en el Parque Citroën en París, Francia (figs. 01 y 02). El Tercer Paisaje es la suma de los espacios donde el hombre deja la evolución del paisaje en manos de la propia naturaleza' ${ }^{12}$. De allí podemos extrapolar que aquello se convierte en un laboratorio donde se puede observar el concepto de autopoiesis, es decir la tendencia que tiene la vida de crear condiciones propicias para su perennidad.

EL TERCER

PAISAJE Y EL

JARDÍN EN

MOVIMIENTO
Desde sus primeros estudios en la isla de Vassivière en el Limousin en Francia (fig. 03) en 1986 para la construcción del Centro de Arte y Paisaje con Aldo Rossi y Xavier Fabre, hasta sus primeros escritos ${ }^{13}$, pasando por sus investigaciones en la Vallée y sus realizaciones de parques públicos como el Parque André-Citroën (figs. 01 y 02), Gilles Clément ha defendido un espacio privilegiado donde recibir la diversidad biológica. El espacio entregado a las leyes de la naturaleza considerado como la reserva genética del planeta, aparece como el espacio del futuro. Con ello Clément ponía de manifiesto el valor semántico de la reutilización de los desechos naturales para abrir una nueva consciencia dentro de la sociedad. Esta visión se ha manifestado de modo singular en propuestas arquitectónicas. Estos conceptos transformaron la percepción de los arquitectos franceses y más precisamente parisinos, a la hora de incluir el elemento "naturaleza", de este nuevo modo, dentro del proyecto arquitectónico. Desde el proyecto de la Fondation Cartier de Jean Nouvel en 1994 hasta la Biblioteca Nacional en París construido por Dominique Perrault en 1995, la nueva relación del hombre con la naturaleza defendida por Gilles Clément abrió una nueva línea de alianza entre arquitectura y naturaleza. Estos dos proyectos desafiaban con sus soluciones técnicas, en el empleo o disposición de la naturaleza, la relación mantenida por entonces entre ambas. Gracias a la inclusión del ingeniero agrónomo ${ }^{14}$ por una parte y de este nuevo jardinero ${ }^{15}$ por otra, en el modo de pensar el proyecto arquitectónico, los arquitectos parisinos empezaron a utilizar la naturaleza como material para la construcción del tiempo ${ }^{16}$ y por lo tanto del cambio, de la transformación, de la mutación, de la evolución ${ }^{17}$ en sus edificios. Aquello daba lugar a un nuevo modo de pensar, no solo las formas en las que la naturaleza se expresa frente al artificio sino la redefinición de los tiempos y formas de organización que le pertenecen: el tiempo y la contingencia formaban parta de la idea de proyecto.

Los conceptos de Gilles Clément, quieren transcender la relación del hombre con la naturaleza: ir más allá de una naturaleza decorativa y recreativa heredada del movimiento higienista del siglo XIX. El jardín en movimiento, Jardin en mouvement, se inspira del espacio sin cultivar, abandonado donde, al no estar restringido por un principio cultural privilegiando el aspecto formal del mundo natural, la naturaleza puede manifestar sus energías, sus crecimientos, sus desplazamientos, sus intercambios. En otras palabras, Gilles Clément quiere recordar al ser humano los procesos intrínsecos que contiene la naturaleza. Procesos tales como sus ciclos temporales, sus sistemas de organización y de gestión ligados a su vez al concepto de contingencia ${ }^{18}$. Una visión mucho más biológica y menos cultural de la que quizá el hombre se puede inspirar para 


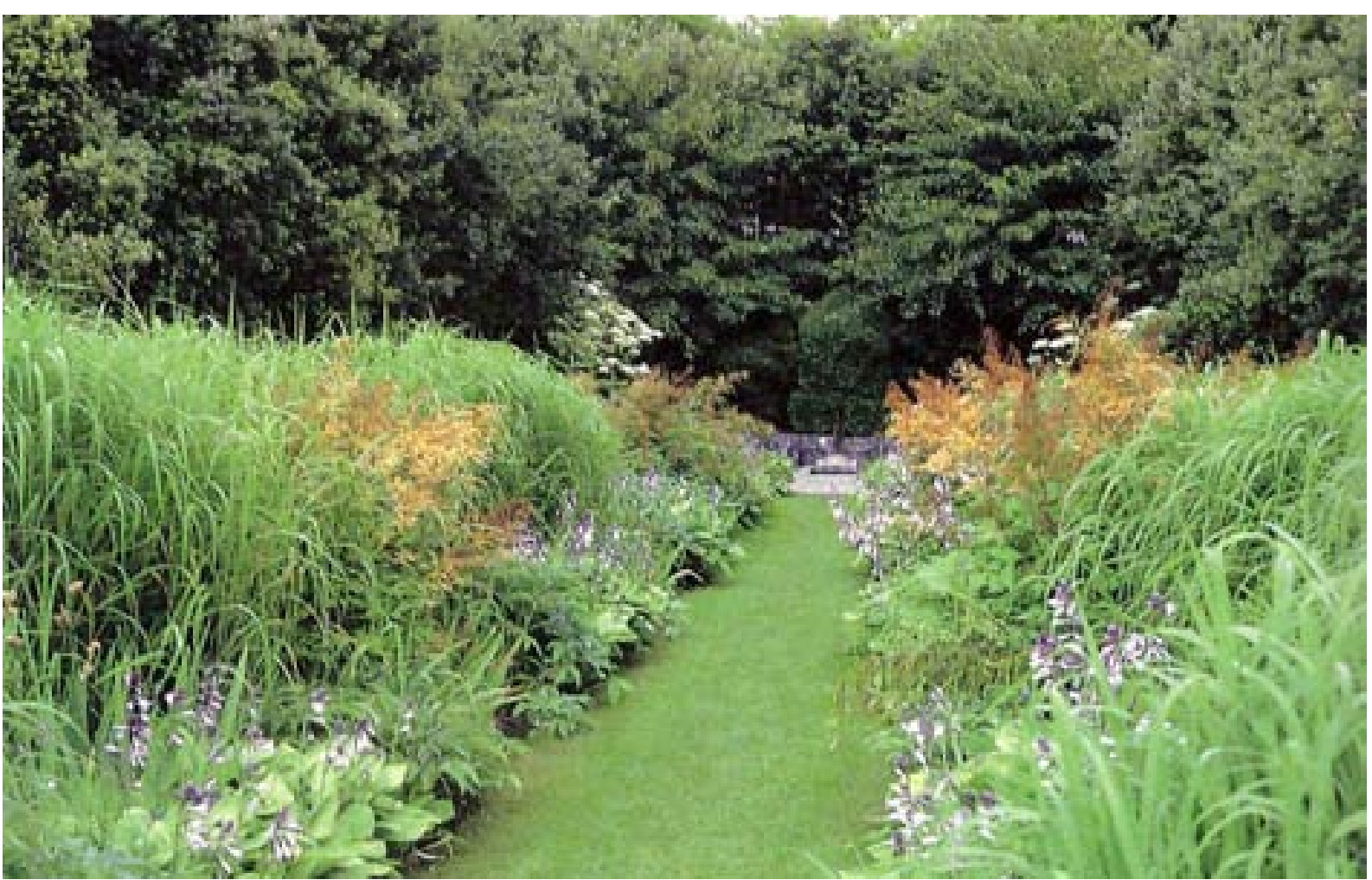

02

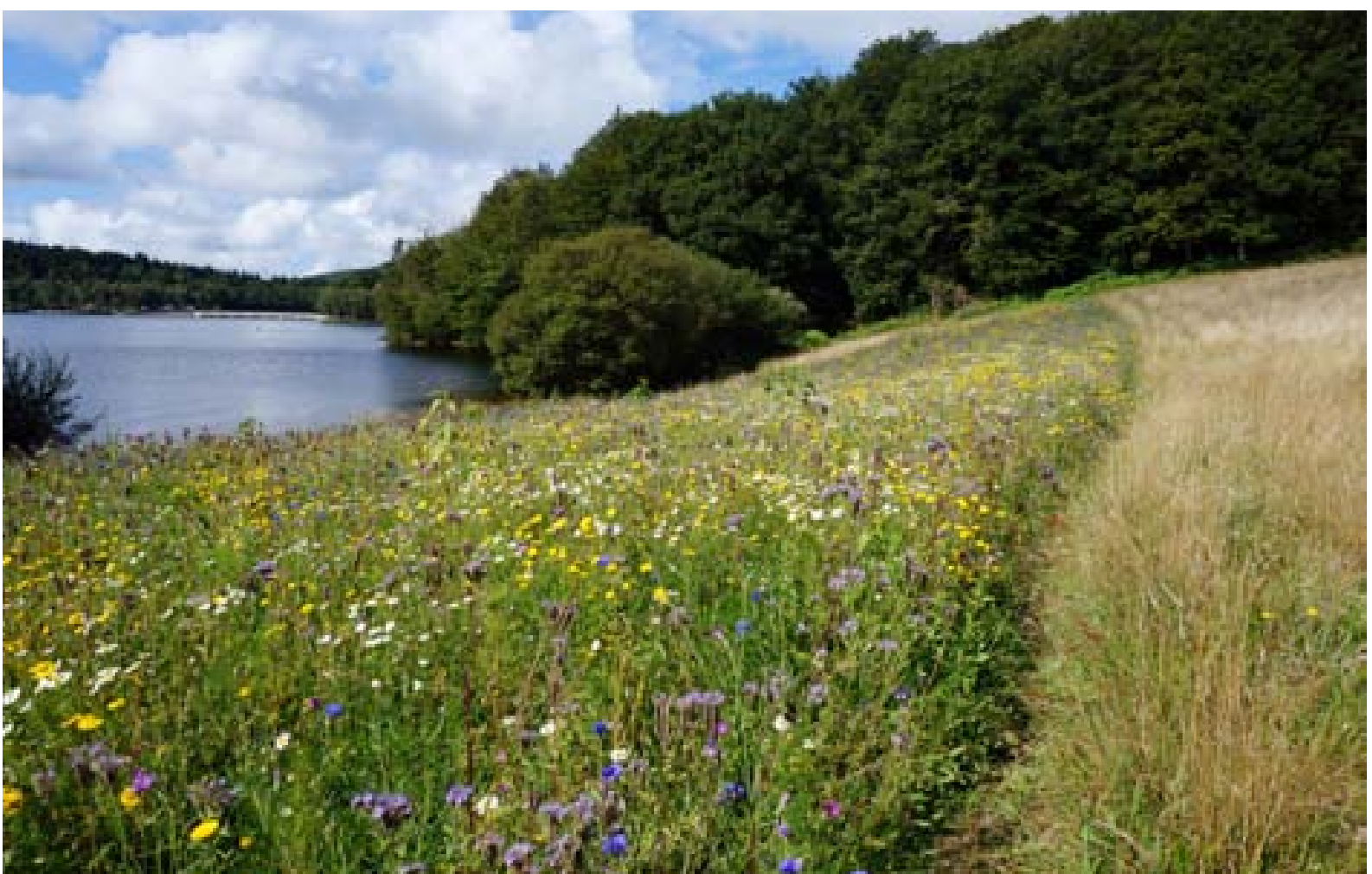




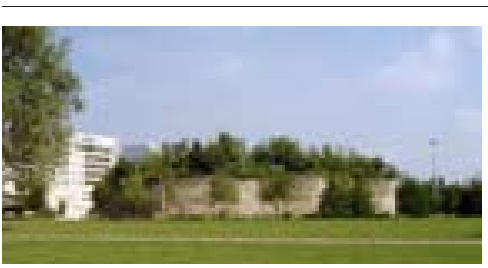

04

Fig. 04

Gilles Clément, parc Matisse, Lille, L'île Derborence et le Boulingrin.

observar más y actuar menos. Este es ahora un jardín espontáneo, que atendido por la mano del hombre, quiere ser testigo o laboratorio donde aprender de sus procesos y restablecer ciclos temporales en la ciudad. Quizá uno de los elementos más valiosos de la postura de Gilles Clément resida en la manifestación de las contingencias y texturas temporales de la naturaleza. Pensamos que es este el elemento que ha influido en cuanto a la descripción y consideración del proyecto arquitectónico.

Aquella postura del jardinero francés desarrollada en los años 90, también se vio inspirada por otra gran esfera de la sociedad. Esta, más intelectual, quedaba representada por filósofos como Michel Serres ${ }^{19}$, Gilles Deleuze ${ }^{20}$ y Félix Guattari ${ }^{21}$ en: la naturaleza como material de "construcción de pensamiento" y de toma de consciencia en la relación del hombre con ella.

El ejemplo de la estructura rizomática que presentaron Deleuze y Guattari22 promovió un re-pensar profundo de cómo organizar la sociedad en aquellos años post mayo de 1968. Desde entonces hasta hoy, vemos cada vez más realizados dichos conceptos en ciertas organizaciones de nuestra sociedad. El valor de la solidaridad y de estructuras menos jerarquizadas a favor de una mayor colaboración tienden a querer ser parte de una nueva manera de organizarse. La observación de la naturaleza se ha convertido en un material de construcción filosófico. Podemos nombrar quizá un hecho constitutivo como el de la organización llamada Friends of Earth², iniciado por David Brower en 1969 en San Francisco en Estados Unidos. Aquella federación de ciudadanos, sensibles a cuestiones medioambientales, defensores de movimientos sociales, desarrolló en los años 1970 una red mundial de ecologistas. Esta estructura solidaria determinada a organizar un sistema agroecológico en varios países fue basada en conceptos de alianza, multiforme y proveedora de interconexiones capaces de explorar las sinergias entre diversos agentes. Esta organización ha demostrado poder enfrentarse a nuevos desafíos y adaptarse a nuevos contextos. Hoy, presentes y activos en 77 países, cuentan con más de 2 millones de miembros. Su expansión y perennidad se debe a una política que privilegia autonomía y autodeterminación. Estos sistemas de gestión, de organización, de mutación, de regeneración son modelos inspirados de la biología que contemplan el parámetro tiempo.

EL PARQUE

MATISSE: LA ISLA

DE DERBORENCE,

LILLE, FRANCIA,

1990-1995
Para ilustrar el argumento del artículo que defiende la naturaleza como material de construcción "de pensamiento en el campo de la arquitectura y la ciudad", utilizaremos el parque Matisse desarrollado por Gilles Clément y la Biblioteca Nacional de Francia en París de

Dominique Perrault. El parque Matisse se llevó a cabo dentro de un proyecto de mayor envergadura llamado Euralille y supervisado por Rem Koolhaas (fig. 04). El terreno dónde se desarrolla el parque representa alrededor de 8 hectáreas y es consecuentemente residual a diferentes proyectos: las vías férreas del tren de alta velocidad que conecta Lille con capitales europeas como Londres, París y Bruselas, un centro comercial, una línea de tranvía, un bloque de viviendas y varias torres para la reconquista de tejido urbano.

La voluntad de crear un espacio forestal inaccesible al público, pero garante de un bioma, consideraba por entonces el bosque 
cómo el futuro de la civilización. La naturaleza salvaje, encerrada en la isla de Derborence, aparece en el proyecto como primer ejemplo de una nueva tendencia que pretendía introducir el bosque en el tejido urbano.

En 1990, Gilles Clément propone la constitución de un símbolo fuerte, un manifiesto in vivo para el hombre del futuro: el bosque, un santuario forjado por el tiempo y la historia ${ }^{24}$. Para ello, el perímetro de la isla sería construido con muros encofrados, hechos de hormigón y desechos directamente recogidos de las obras de excavación para la estación de tren. Allí se auto cultiva un bioma inaccesible al público.

Con este modelo, en pleno tejido urbano, la isla se convierte en un fragmento del Tiers-paysage o Tercer paisaje.

Por otra parte el uso de unos pinos silvestres trasplantados en el corazón de París para organizar la Biblioteca Nacional de Francia, ofrece un bosque artificial que permite contemplar y recordar la fragilidad de la naturaleza. Su papel simbólico, ya no solo decora o recrea si no instruye, inspira un colectivo de investigadores capaces de aportar nuevos sistemas de pensamientos. Detrás de aquella propuesta provocadora, estaba el desafío técnico asumido por el ingeniero agrónomo Erik Jacobsen, responsable del trasplante de arboles adultos de 40 años de edad. La propuesta ganadora del concurso del arquitecto Dominique Perrault, parece directamente influenciada por los trabajos de Gilles Clément. Perrault utiliza la construcción de un bosque artificial como un paradigma que representa el saber de la humanidad, inaccesible para su preservación pero capaz de influir los modos de pensar de los investigadores que acuden a la biblioteca ${ }^{25}$.

ECOLOGÍA

TEMPORAL: LA

AGROFORESTERÍA,

UN CAMBIO DE

PARADIGMA
El conocimiento del hombre construido desde la observación y conceptualización del medio forestal, constituye un corpus llamado agroforestería. Dentro de la línea del biomimetismo, la agroforestería o agrosilvicultura, planteada a escala de ciudad, parece imponerse tanto como

ecosistema (con el que trabajar y convivir) así como modelo de organización. Es decir, si pensamos la arquitectura como un sistema abierto que intercambia, de manera optimizada, energía, materia e información, cabría entender y representar la arquitectura desde tres niveles: la forma, el proceso y el sistema.

Desde esta posición, la arquitectura, debería representar ciclos temporales más amplios que los que condicionan su construcción. Diseñar también la vida del edificio, como un organismo vivo, con el fin de anticipar mutaciones venideras, así como permitir su cooperación o alianzas dentro de una red más amplia de ecosistemas múltiples que componen su contexto local ${ }^{26}$.

Por esta razón, este artículo defiende una postura no exclusivamente literal de la naturaleza como material de construcción si no también simbólica, semántica y filosófica. Redefinir la relación que tenemos con la naturaleza que nos envuelve, es quizá entender primero que tenemos que reconsiderar por completo los ciclos temporales que conforman nuestro presente. Según el teórico y urbanista Paul Virilio, el desarrollo de las tecnologías nos ha sometido a una aceleración del tiempo mundiali27. Quizá el hecho de considerar la naturaleza como material de construcción en arquitectura implica reconsiderar los ciclos temporales para reaprender a vivir con mayor contingencia el espacio doméstico y urbano. 


\section{Fig. 05}

Reconstitución artística de una escena de arada y siembra en el Antiguo Medio Oriente a principios del $8 \mathrm{vo}$ milenario.

\section{Recientemente, Keller Easterling, en su conferencia ${ }^{28}$} Medium Design, a propósito de la construcción del medio, plantea redefinir la representación del tiempo en arquitectura y en la ciudad. La autora presenta las ventajas del uso de la herramienta contemporánea del time-lapse document para representar la contingencia en arquitectura. El objetivo de dicho documento permitiría abordar ciclos de mayor escala ausentes en los documentos convencionales que definen los edificios o las ciudades.

A partir del Neolítico, el Homo Sapiens observa los diferentes ciclos de los vegetales con el fin de poder domesticarlos progresivamente y desarrollar la agricultura (fig. 05).

El conocimiento de los ritmos de los seres vivos dio pie a una ciencia llamada cronobiología. Dicha ciencia que observa y analiza los elementos sincronizadores y de-sincronizadores dentro de los ciclos evolutivos de la naturaleza integra un sistema de pensamiento llamado ecología temporal ${ }^{22}$. Esta ciencia reconoce y defiende determinados ciclos temporales que le son propios a sistemas vivos, que los organizan y determinan su perennidad.

El reconocimiento del parámetro tiempo se ve defendido en el símbolo "bosque" por parte de Gilles Clément. El concepto de Tercer Paisaje pone de manifiesto una reconsideración temporal dentro de la construcción del medio urbano. Como hemos visto previamente, la observación del bosque como sistema puede ayudarnos a transformar nuestros paradigmas en la disciplina arquitectónica: energía, materia, información y contingencia. Además de controlar parámetros atmosféricos,

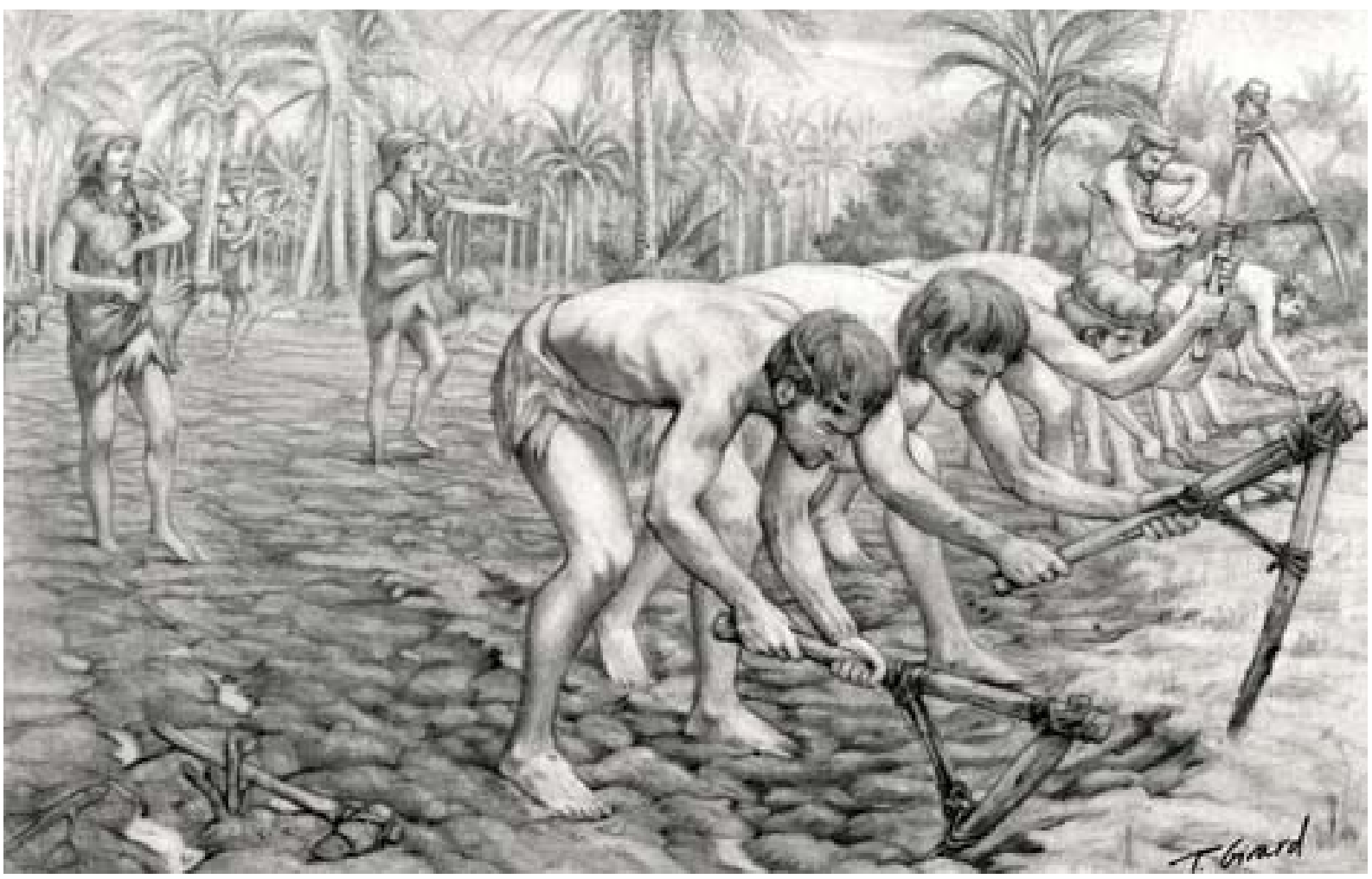




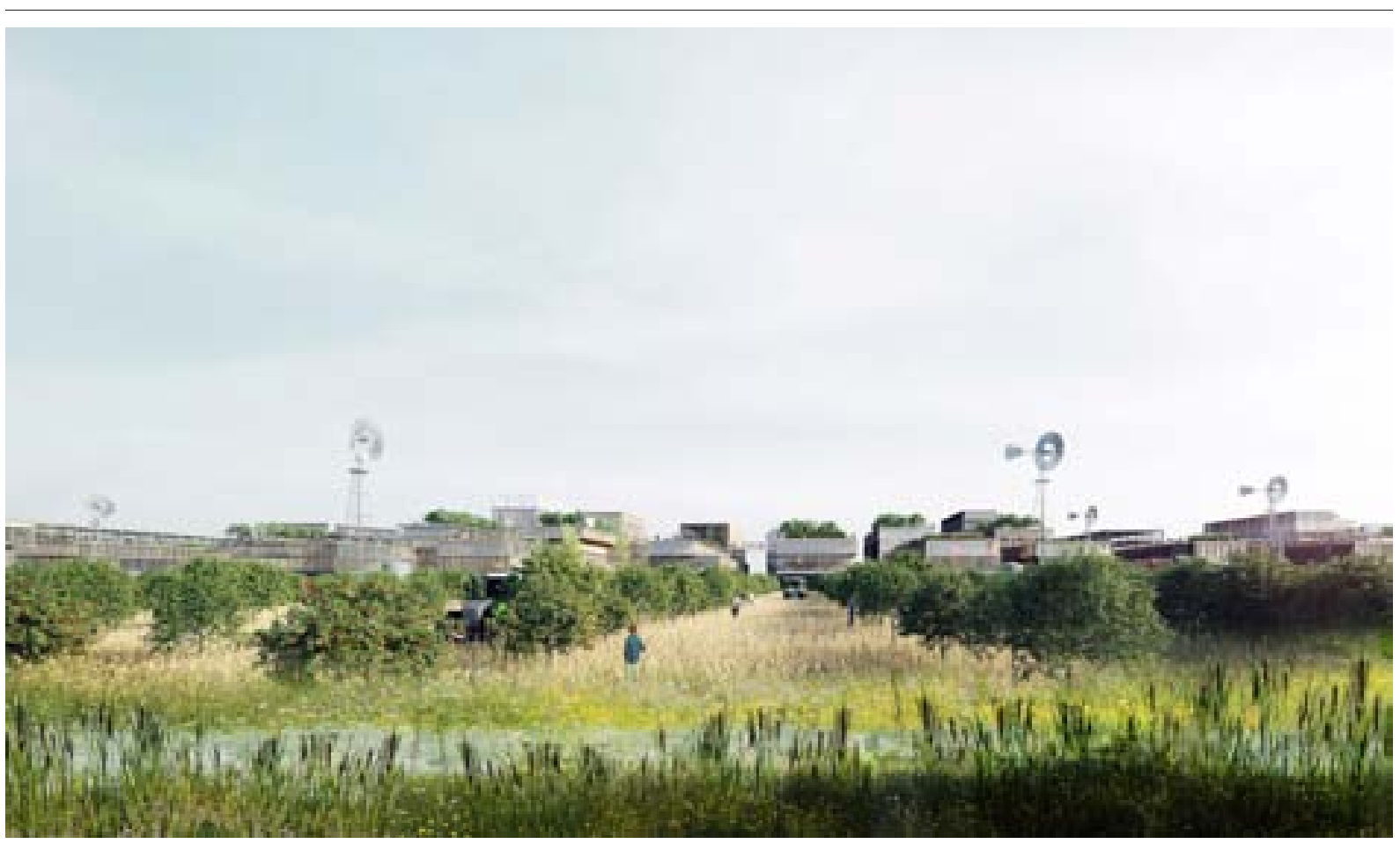

06

proteger el suelo y regular la red de aguas ${ }^{30}$, el bosque juega un papel no menos importante sobre el bienestar del hombre. El sistema temporal del bosque podría ser un regulador o sincronizador para el ciclo temporal del ciudadano: tiempo individual y social. Consecuentemente, el bosque podría regular su estado psicofísico. Un estado que podría a su vez cultivar una mayor harmonía o sinergia en la sociedad.

Este bien natural representa una apuesta económica muy importante y ha sido reconsiderada en las estrategias de desarrollo del territorio, desde hace más de una década. Por ejemplo, el bosque es un recurso natural capaz de jugar el papel de los ciclos productivos para la alimentación, la energía y la industria de la madera como material. Por razones, fisiológicas, medioambientales y económicas, el mundo de la agrosilvicultura se ha convertido en un elemento fundamental en las estrategias políticas actuales en países tales como Francia ${ }^{31}$.

La introducción del bosque en la civilización urbana planteada en 1968 por Yves Bétolaud ${ }^{32}$ (1926-2003) ingeniero rural, de aguas y de bosques en París parece predecir lo que estamos viviendo hoy en París, Francia, con el proyecto Agriville propuesto por el grupo constituido por tres estudios de arquitectura, agence XTU, Arep y Jean-Paul Viguier \& Associés (fig. 06).

Tal y cómo lo presentaba el ingeniero agrónomo francés el bosque empieza a imponerse como equipamiento urbano al mismo nivel que una escuela, un hospital o una biblioteca. Podríamos decir que el bosque educa, sanea e instruye además de poder alimentarnos y abastecernos de energía. El deseo por la naturaleza en los contextos urbanos y en el espacio doméstico parece también haber generado otros problemas del que nos advierte Paul Arnould ${ }^{33}$, geógrafo y biogeógrafo francés. Nos recuerda que el modelo autopoiético de la naturaleza salvaje conlle-
Fig. 06

Fotomontaje para "Inventons la Metropôle du Grand Paris", proyecto Agriville, Gonesse Noreste de París. 
va problemas específicos del mundo de los seres vivos: la naturaleza no sabe de límites. Pero también advierte que la naturaleza planificada está fuertemente endeuda con la tecnología y las energías fósiles. El ejemplo de las cubiertas vegetales, fachadas vegetales y orillas de ríos recalificadas conllevan otro tipo de complejidades sistémicas tales como proliferaciones animales menos deseables como son las cucarachas, las ratas o los mosquitos, que son síntoma del desajuste de equilibrios que requieren muchos otros parámetros y factores. El desarrollo de la naturaleza dentro de la ciudad requiere como primer factor una concepción técnica del tiempo que defiende la ecología temporal. Ésta es un factor que entre otros permite ralentizar la degradación de la energía del sistema y con ello hacer factible la convivencia entre lo natural y lo artificial.

LA ECOLOGÍA DESDE UNA CONCEPCIÓN TÉCNICA
En 1968 llya Prigogine demostró que todo sistema abierto debe considerar una parte de entropía a un punto de inflexión a partir del cual el sistema puede evolucionar a un modo caótico, o auto organizarse alrededor de un nuevo equilibrio complejo. Por esta razón, la técnica

aparece como herramienta capaz de ralentizar el nivel de entropía al que tiende a llegar rápidamente la convivencia entre arquitectura y naturaleza.

Como vamos viendo, la construcción de nuevos paradigmas inspirados por la naturaleza y más específicamente aquellos que se apoyan en los sistemas internos que organizan los bosques, no podrá hacerse sin consideraciones técnicas desarrolladas en varias direcciones.

Si se quiere entender el modo en que la técnica se presenta como vehículo para el cambio de paradigma del hombre con la naturaleza expresada en los bosques, debemos presentar el significado de la técnica en general y más específicamente dentro del mundo agroforestal.

Gilbert Simondon en Du mode d'existence des objets techniques, defiende que la técnica no es un conjunto de medios o herramientas, si no un modo d'être-au-monde o de ser en el mundo: una fase de la cultura ${ }^{34}$. El filósofo afirma que para entender sus fracasos, la técnica busca sus verdaderos valores de fondo y es así cómo produce la ciencia ${ }^{35}$. En otras palabras, proyectándolo en el caso que nos ocupa, la agroforestería o agrosilvicultura, como técnica, sería el modo técnico que reúne todo el conocimiento del hombre sobre el manejo de los bosques. La ciencia agroforestal, consecuente del conocimiento adquirido por el hombre a través de su observación y conceptualización de dicho sistema biológico, permite al hombre desarrollar objetos técnicos capaces de ralentizar la degradación de la energía contenida intrínsecamente en el sistema "bosque" ${ }^{36}$. El objeto técnico, según las definiciones de Simondon, representa tanto un saber concreto como abstracto de un campo determinado. El artesano, o jardinero forestal, mantiene una relación concreta entre el mundo y el objeto técnico. Sin embargo el objeto pensado por el ingeniero es un objeto técnico abstracto, desligado del mundo natura| ${ }^{37}$. La técnica ha de ser reconocida desde dos ángulos a veces opuestos: la técnica con un estatuto de mayoridad y el de minoridad ${ }^{38}$. Siguiendo la definición de Simondon, la primera sería la del ingeniero, la segunda la del artesano. Los dos son los vínculos o transmisores del conocimiento de dicho campo al resto de la sociedad. Cada uno de ellos representa, de manera muy diferente, esta técnica y contribuye de manera diversa a que la técnica pueda incorporarse a la cultura. 
Según el filósofo francés, el progreso técnico en última instancia consiste en ralentizar la entropía contenida en cualquier sistema del universo. Por lo tanto la técnica, en su fin último, se presenta al hombre como el medio de gestión de los ciclos temporales contenidos en los sistemas.

La consideración técnica del campo agroforestal es capaz de actuar como mediador ante la degradación de la energía contenida en el sistema bosque siempre y cuando el ingeniero agrónomo y el jardinero forestal sean integrados dentro de la función social, y no solo en relación con el conocimiento científico de la naturaleza.

Desde la perspectiva que propone este artículo, ellos, son los actores capaces de permitir la convivencia de lo natural con lo artificial. Esta relación está sujeta a muchas disfunciones o degradación de la energía como ha señalado Paul Arnould ${ }^{39}$. Este enunciado se hace visible a través de problemas tangibles como la aparición de animales, plantas en sobre abundancia, invasivas o pandemias que llevan a la muerte de ecosistemas urbanos. Por lo tanto si la consideración de la naturaleza como materia de construcción de pensamiento en arquitectura nos ayuda a redefinir relaciones temporales con los elementos componentes de nuestro medio, la técnica se impone como el elemento gestor de la degradación de la energía del sistema.

Aquí quisiéramos defender el bosque como materia de construcción de nuestras ciudades desde un punto de vista biológico y cultural. El medio forestal aparece como un elemento sincronizador y socializador. Como hemos dicho, un ecosistema que debe ser considerado como equipamiento urbano a la altura de una biblioteca, una escuela o un hospital. El bosque permitiría introducir ciclos temporales paradigmáticos dentro de la ciudad. Sin embargo advertimos que esto sólo será factible desde una revalorización de la técnica. Ella media entre el hombre y la naturaleza. El bosque junto con la técnica agroforestal, transmitida por el ingeniero agrónomo y el jardinero, podrían potenciar el concepto de contingencia en nuestro medio urbano.

El desarrollo de la ciudad así como los proyectos arquitectónicos que participan de su crecimiento, deberían reconsiderar el papel de estos actores dentro de la definición de nuevas propuestas.

CONCLUSIÓN

Para realizar "la naturaleza como material de construcción del pensamiento" y por tanto "pensamiento arquitectónico", necesitamos urgentemente una arquitectura interpretada desde un sistema dinámico y dialéctico que reintegra el tiempo y la incertidumbre, consubstanciales a la naturaleza ${ }^{40}$. La naturaleza, representada por un ecosistema como el bosque, debe recordar y llegar a imponer el concepto de contingencia al hombre. El bosque es en esencia el resultado de una confrontación entre restricciones naturales e intervenciones humanas ${ }^{4}$. El ecosistema forestal es fruto de la contingencia y por lo tanto es capaz de simbolizarla ante la sociedad (fig. 07).

La convivencia entre un sistema vivo y un sistema regido por otras leyes organizadoras como el medio urbano, requiere una reconsideración de nuestra relación con la técnica. Ésta debería de ser asimilada por la cultura y por ello representada en las propuestas arquitectónicas y urbanas. 
Fig. 07

Biblioteca Nacional de Francia, arquitecto Dominique Perrault, París, Francia.
Gracias a la técnica y a sus agentes transmisores como son el ingeniero agrónomo y el jardinero forestal, podremos integrar la naturaleza como material de construcción, en un sentido literal. Gilles Clément, defensor del jardín en movimiento y del Tercer Paisaje, es consciente que la mano del hombre es la que modela día tras día, organiza, y gestiona un sistema "natural". La intervención del hombre, dentro de un concepto de una ecología temporal y técnica, podría ser considerada como una contribución a la reducción de la degradación de la energía del sistema pero sin alterar su evolución. La naturaleza tiende a crear condiciones propicias a su perennidad pero a su vez todo sistema conlleva una parte de degradación de energía. La relación de contingencia que debe mantener el hombre con el ecosistema forestal implantado en un contexto urbano se convierte también en un recurso intelectual y cultural. Por esta razón, el artículo toma ventaja del título original de la convocatoria, en ir más allá considerando la naturaleza como material de construcción "de modos de pensar, organizar y relacionar".

En otras palabras, la observación de la naturaleza permite construir un corpus de conocimiento que influye tanto sobre nuestro medio físico como cultural. Aquel proceso de tecnificación de la naturaleza nos ayuda a re-conceptualizar sistemas organizativos, sistemas relacionales a través de conceptos de mutación, de autopoeisis o exaptación ${ }^{42}$, proveyendo de nuevas direcciones, fuentes de inspiración para el desarrollo de la sociedad y por lo tanto de su hábitat.

La naturaleza más allá de lo que puede producir, sanear y recrear es un libro manifiesto in vivo, en equivalencia una obra filosófica de Gilles Deleuze, del que nos podemos inspirar. La contingencia presente en el mundo de los seres vivos es un símbolo fuerte a reconsiderar en nuestro cotidiano. Aunque la naturaleza de apariencia salvaje, requiera ser controlada dentro de la ciudad, sus ciclos temporales siguen transcendiendo sobre el estado psicofísico del ciudadano. El jardín de la Fundación Cartier ideado por el artista Lothar Baumgarten en 1992 trabaja con el espacio vivo. El jardín, aparentemente salvaje, integra la noción de tiempo y utiliza los ciclos temporales como material de construcción gracias al sabio control del jardinero. La transmisión de los procesos de transformación de la naturaleza a lo largo del tiempo permite al ciudadano experimentar su potencial de contingencia. La naturaleza asume el accidente, lo imprevisto que favorece la construcción de lazo social e individual.

El bosque, pues, debería de ser considerado no sólo como un equipamiento natural también cultural que impulsa en varias direcciones nuestra manera de relacionarnos individualmente, socialmente, con él y con la naturaleza en general.

La exigencia urbana de actuaciones continuas sin margen y sobre todo soporte, provoca la negación del tiempo de los espacios y por lo tanto de sus actores ${ }^{43}$. Por esta razón, queremos defender el reconocimiento del sistema agroforestal desde su presencia física en el entorno urbano y del proyecto arquitectónico así como el valor de los actores que participan de su existencia y permanencia. Estos dos elementos permitirían considerar la naturaleza como material de construcción de nuevos paradigmas en el campo de la arquitectura y de la ciudad: el tiempo y la contingencia. RA 


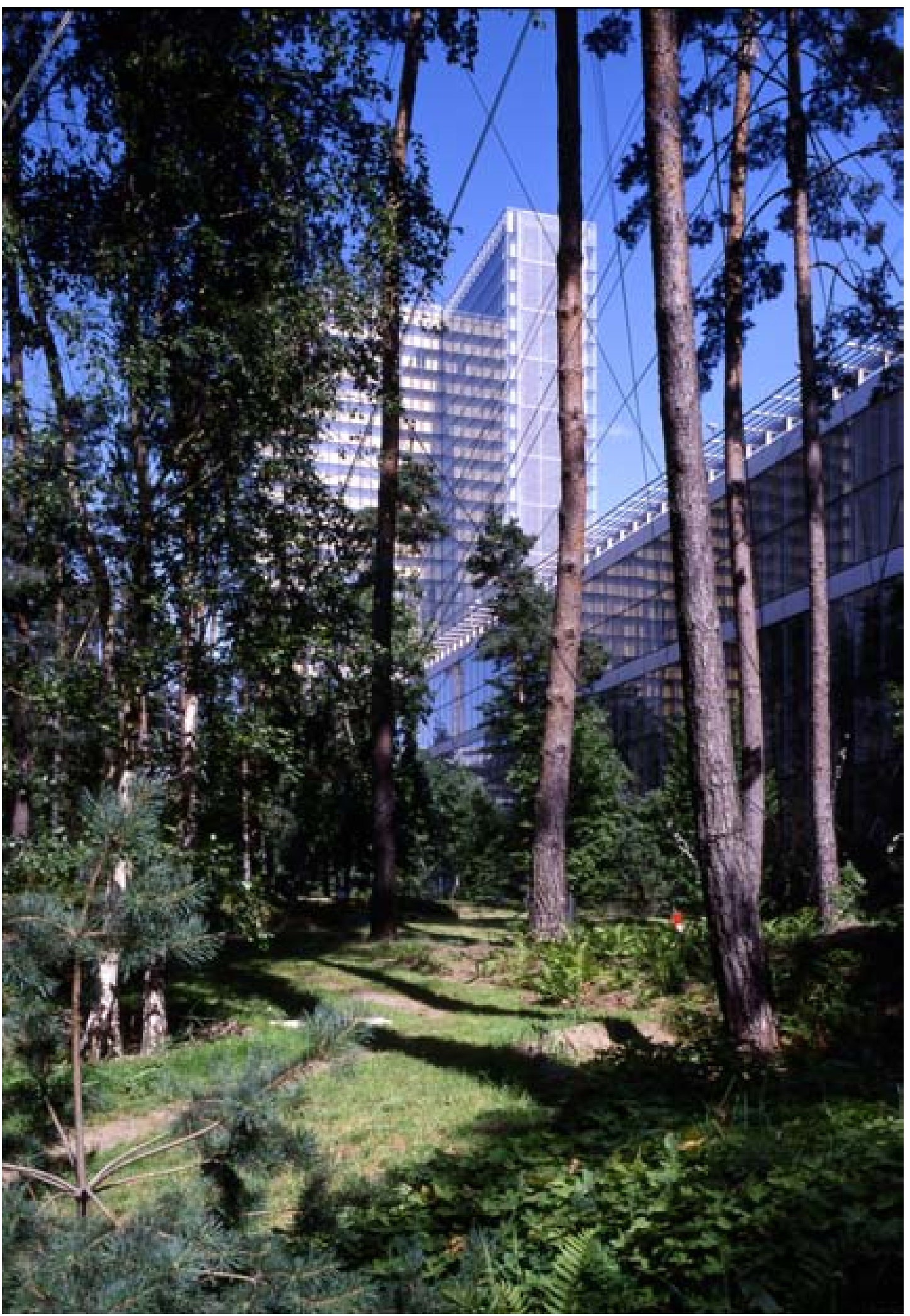


01. DELANNOY, Emmanuel, Dictionnaire de la pensée écologique sous la direction de Dominique Bourg et Alain Papaux, Edición PUF 2015, p. 88.

02. SERRES, Michel, Le contrat naturel, Paris, François Bourin, 1990.

03. En 1972, los biólogos chilenos Humberto Maturana y Francisco Varela propusieron este concepto para definir la química de auto-mantenimiento de las células vivas. La autopoiesis es la tendencia que tiene la vida de crear condiciones propicias para su perennidad.

04. GOULD, S. J. y VRBA, E. S. "Exaptation - a missing term in the science of form". Paleobiology, 1982.

05. SIMONDON, Gilbert, Du mode d'existence des objets techniques, Paris Aubier, 2012.

06. CANS, Roger, Dictionnaire de la pensée écologique sous la direction de Dominique Bourg et Alain Papaux, Edición PUF 2015 pp. 322-325.

07. PAQUOT, Thierry, Dictionnaire de la pensée écologique sous la direction de Dominique Bourg et Alain Papaux, Edición PUF 2015, pp. 144-147.

08. En 1971, Se crea el Centre National d'Étude et de Recherche du Paysage CNERP encargado de promover una nueva educación entorno al Paisaje a través del desarrollo de estudios a escala territorial así como la formación de altos cargos administrativos y técnicos. En 1977 se crean los CAUE que significa Conseils d'Architecture, d'Urbanisme et d'Environnement traducido como Consejos de Arquitectura, Urbanismo y Medio Ambiente que permitieron establecer lazos, desde entonces inalterables, entre arquitectura, urbanismo y paisaje. Estos consejos promovieron nuevos valores paisajísticos dentro del POS, Plan de Ocupación de Suelos. En 1977, una ley sobre la Arquitectura rige los principios de las CAUE La création architecturale, la qualité des constructions, leur insertion harmonieuse dans le milieu environnant, le respect des paysages naturels ou urbains ainsique du patrimoine sont d'intérêt public. [...] Des Conseils d'architecture, d'urbanisme et d'environnement sont institués. Datos recogidos de Carnet de Recherches du Comité d'Histoire du Ministère de la Culture sur les politiques, les institutions et les pratiques culturelles escrito por Pascal Desvaux https://chmcc.hypotheses.org/4051.

09. En 1971, nace el Ministerio de Medio Ambiente bajo la dirección de Pierre Poujade. En Francia, durante los años 1970 se empieza a legitimar la defensa de la naturaleza: René Dumont obtiene la primera candidatura a la elección presidencial de 1974. Sin embargo hubo que esperar a los años 1980 para ver una representación contundente de una política ecologista gracias a Brice Lalonde en la presidencial de 1981 que obtuvo más de un millón de votos. En 1985, la dirección de la Arquitectura se fusiona con la Dirección de Urbanismo y Paisajes.

\section{SOULIÉ, Charles, Le destin d'une} institution d'avant-garde: Histoire du department de Philosophie Parix VIII, Histoire de léducation, n. 77, janvier 1998, pp. 47-69.

11. DPLG significa Diplômé par le Gouvernement traducido como diplomado por el Gobierno.

12. CLÉMENT, Gilles, JONES, Louisa, Gilles Clément une écologie humaniste, Aubanel, Genève, Suisse, 2006

13. CLÉMENT, Gilles, La friche apprivoisée, Urba 209, septiembre 1985

14. NOUGARĖDE, Olivier, ALPHANDÉRY, Pierre, le silvarium de la Grande Bibliothèque, INRA, Economie et Sociologie rurales, unité STEPE, Courrier de l'Environnement de l'iNRA n" 24. Extraido del artículo publicado en Arbre Actuel, n'18, avril-mai 1995, pp. 16-20, Le cloître forestier de laBibliothèque nationale de France.

15. MOSBACH, Catherine, CLARAMUNT, Marc, La nature des interventions paysagères. Exemples de créations et de transformations, In: Les Annales de la recherche urbaine, $N^{\circ} 74$, 1997. Natures en villes. pp. 137-142.

16. Ibidem.

17. NOUGARĖDE, Olivier, ALPHANDÉRY, Pierre, le silvarium de la Grande Bibliothèque, INRA, Economie et Sociologie rurales, unité STEPE, Courrier de l'Environnement de l'iNRA n" 24. Extraido del artículo publicado en Arbre Actuel, n'18, avril-mai 1995, pp. 16-20, Le cloitre forestier de laBibliothèque nationale de France.
18. ARNOULT, Paul, Unjardin dans la ville, quelle biodiversité urbaine pour demain? L'exemple de Gilles Clément à L'ENS de Lyon, artículo "Environnement Ville Société", 2012

19. SERRES, Michel, Le contrat naturel, François Bourin, Paris 1990.

20. DELEUZE, Gilles, GUATTARI, Félix, Capitalisme et Schizophrénie 2. Mille Plateaux. Editions de Minuit, Paris, 1980.

21. GUATTARI, Félix, Les trois écologies, Galilée, Paris, 1989.

22. DELEUZE, Gilles, GUATTARI, Félix, Capitalisme et Schizophrénie 2. Mille Plateaux. Editions de Minuit, Paris, 1980.

23. CHARTIER, Denis, Dictionnaire de la pensée écologique sous la direction de Dominique Bourg et Alain Papaux, Edición PUF 2015, pp. 17-19.

24. CLÉMENT, Gilles, JONES, Louisa, Gilles Clément une écologie humaniste, Aubanel, Genève, Suisse, 2006 pp. 140-147.

25. NOUGARĖDE, Olivier, ALPHANDÉRY, Pierre, le silvarium de la Grande Bibliothèque, INRA, Economie et Sociologie rurales, unité STEPE, Courrier de l'Environnement de l'iNRA n. 24. Extraido del artículo publicado en Arbre Actuel, n. 18, avril-mai 1995, pp. 16-20, "Le cloître forestier de laBibliothèque nationale de France".

26. WILES, Graham "Cardboard to Caviar". Graham Wiles conceived a lucrative cycle of processes, demonstrating the 'closed-loop' model. He identified a path of operations emerging from a common waste product (cardboard boxes) and turning it into a high value end product (caviar), which could be sold back to the original producer of waste.

27. VIRILIO, Paul, El cibermundo, la política de lo peor, Eds. Cátedra, Madrid, España, 1997.

28. EASTERLING, Keller, Medium Design, Strelka, 2018.

29. PAQUOT, Thierry, Dictionnaire de la pensée écologique sous la direction de Dominique Bourg et Alain Papaux, edición PUF 2015, pp. 346-349.

30. BÉTOLAUD, Yves, Forêts et civilisation urbaine, Paris, 1968 accesible http:// documents.irevues.inist.fr 


\section{Referencias bibliográficas}

31. En el 2008, el Consejo general de agricultura, alimentación y espacios rurales en Francia presenta un ensayo prospectivo sobre los bosques franceses de cara a 2050-2100 con varios escenarios de desarrollo del parque forestal en el territorio.

32. BÉTOLAUD, Yves, Forêt et civilisation urbaine, Class. Oxford 907, Paris, 1968, pp. 535-545.

33. ARNOULD, Paul, et al., La Nature en ville: l'improbable biodiversité, Géographie, économie, société 2011/1 (Vol. 13), pp. 45-68.

34. BONTEMS, Vincent, Dictionnaire de la pensée écologique sous la direction de Dominique Bourg et Alain Papaux, Edition PUF, Paris, 2015, p. 935.

35. lbídem, p. 935.

36. SIMONDON, Gilbert, Du mode d'existence des objets techniques, Editions Aubier, Paris, 2012

37. Ibídem p. 126.

38. Ibídem p. 123.

39. ARNOULD, Paul, La Nature en ville: l'improbable biodiversité, Géographie, économie, société 2011/1 (Vol. 13), pp. 45-68.

40. BOYER R.; CHAVANCE B., GODARD O., Les figures de l'irréversabilité en économie, Paris EHESS, 1991.

41. ARNOULD, Paul, Un jardin dans la ville, quelle biodiversité urbaine pour demain? L'exemple de Gilles Clément à L'ENS de Lyon, artículo "Environnement Ville Société", 2012.

42. DELANNOY, Emmanuel, Dictionnaire de la pensée écologique sous la direction de Dominique Bourg et Alain Papaux, Edición PUF 2015, p. 91. El concepto de exaptación utilizado en biologia se refiere a cómo los organismos vivos en determinados contextos utilizan órganos preexistentes de un modo diferente du su estado original.

43. MOSBACH, Catherine; CLARAMUNT, Marc, La nature des interventions paysagères. Exemples de créations et de transformations, In: Les Annales de la recherche urbaine, n. 74, 1997, natures en villes, pp. 137-142.
-ARNOULD, Paul, et al., La Nature en ville: l'improbable biodiversité, Géographie, économie, société 2011/1 (Vol. 13), pp. 45-68.

-ARNOULD, Paul, Un jardin dans la ville, quelle biodiversité urbaine pour demain? L'exemple de Gilles Clément à L'ENS de Lyon, artículo "Environnement Ville Société", 2012

- BÉTOLAUD, Yves, Forêts et civilisation urbaine, Paris, 1968 accesible http:// documents.irevues.inist.fr

-BONTEMS, Vincent, Dictionnaire de la pensée écologique sous la direction de Dominique Bourg et Alain Papaux, Edition PUF, Paris, 2015, pp. 935.

-BOYER R.; CHAVANCE B., GODARD O., Les figures de l'irréversabilité en économie, Paris EHESS, 1991

- CANS, Roger, Dictionnaire de la pensée écologique sous la direction de Dominique Bourg et Alain Papaux, Edición PUF 2015, pp. 322-325.

- CHARTIER, Denis, Dictionnaire de la pensée écologique sous la direction de Dominique Bourg et Alain Papaux, Edición PUF 2015, pp. 17-19.

- CLÉMENT, Gilles, La friche apprivoisée, Urba 209, septiembre 1985.

- CLÉMENT, Gilles, JONES, Louisa, Gilles Clément une écologie humaniste, Aubanel, Genève, Suisse, 2006.

- DELANNOY Emmanuel, Dictionnaire de la pensée écologique sous la direction de Dominique Bourg et Alain Papaux, Edición PUF 2015, pp. 88-91.

-DELEUZE, Gilles; GUATTARI, Félix, Rhizome, Editions de Minuit, Paris, 1976.

- EASTERLING, Keller, Medium Design, Strelka, 2018.

- GUATTARI, Félix, Les trois écologies, Galilée, Paris, 1989.

- MOSBACH, Catherine, CLARAMUNT, Marc, La nature des interventions paysagères. Exemples de créations et de transformations, In: Les Annales de la recherche urbaine, n. 74, 1997. Natures en villes, pp. 137-142.
- NOUGARĖDE, Olivier, ALPHANDÉRY, Pierre, le silvarium de la Grande Bibliothèque, INRA, Economie et Sociologie rurales, unité STEPE, Courrier de l'Environnement de l'iNRA n. 24. Extraido del artículo publicado en Arbre Actuel, n. 18, avril-mai 1995, pp. 16-20, "Le cloître forestier de laBibliothèque nationale de France".

-PAQUOT, Thierry, Dictionnaire de la pensée écologique sous la direction de Dominique Bourg et Alain Papaux, edición PUF 2015, pp. 144-147.

- SERRES, Michel, Le contrat naturel, Paris, François Bourin, 1990.

- SIMONDON, Gilbert, Du mode d'existence des objets techniques, Paris Aubier, 2012.

-SOULIÉ, Charles, Le destin d'une institution d'avant-garde: Histoire du department de Philosophie Parix VIII, Histoire de l'éducation n. 77, janvier 1998, pp. $47-69$.

- VIRILIO, Paul, El cibermundo, la política de lo peor, Ediciones Cátedra, Madrid, España, 1997.
RA. Revista de Arquitectura Núm. 20 - 2018

P. 54-69 